\title{
Fast complex arm movements in Parkinson's disease
}

\author{
A BERARDElli, N ACCORNERo, M ARGENTA, G MECO, M MANFREdi \\ From Quinta Clinica Neurologica, Centro Disordini del Movimento, Dipartimento di Scienze Neurologiche, \\ Universita' di Roma "La Sapienza", Rome, Italy
}

SUMMARY Fast arm movements involving the shoulder and elbow joints have been analysed in normal controls and in patients with Parkinson's disease. The subjects were requested to draw on a graphic tablet triangles and squares of different size and shape. The patients produced a larger number of EMG bursts compared with controls. The movements were accurate, and each segment of the geometric figures was performed with a roughly straight trajectory, but the time necessary to trace the geometric figures and the pauses at the vertices were prolonged. We conclude that in Parkinson's disease the disability in generating two joint ballistic movements depends on a difficulty in running motor programmes for complex trajectories.

Patients with Parkinson's disease perform ballistic movements slowly at proximal ${ }^{12}$ and distal ${ }^{3}$ joints. Hallett and Khoshbin ${ }^{2}$ who studied elbow movements, have observed that in normal subjects flexion movements were performed with a single triphasic EMG pattern while in patients with bradykinesia the movement required additional cycles of alternating EMG bursts in the biceps and triceps muscles. These abnormalities are present also in movements performed without postural support. ${ }^{3}$ The mechanisms responsible for the bradykinesia are not clear. Using electrical stimulation of the motor cortex through the scalp, the excitability and conduction velocity of the corticospinal pathway proved to be normal in bradykinetic patients. ${ }^{4}$ An explanation can be that in bradykinetic patients the movement signal delivered to the motor cortex is defective.

In this paper we report a study of ballistic arm movements involving the shoulder and elbow joints. The trajectory, the movement duration and the EMG activity from two pairs of agonist and antagonist muscles have been analysed to test whether Parkinsonian patients can plan and perform accurately complex trajectories.

\section{Material and methods}

The study was performed on 12 patients with Parkinson's

Address for reprint requests: Dr Alfredo Berardelli, V Clinica Neurologica, Viale dell'Universita 30, 00185 Roma, Italia.

Received 22 March 1985 and in revised form 1 October 1985. Accepted 20 October 1985 disease aged from 34 to 70 (mean $58 \pm 11$ ). The disease duration varied from 1 to 10 years. All the patients had a moderate to severe degree of bradykinesia and rigidity. A moderate tremor at rest was present in six patients. All the patients were under different drug treatments. The results were compared with a group of 10 age-matched normal controls (mean age $50 \pm 10$ years, range 30 to 60 ) with no history of neurological disease.

The subjects sat in a chair with the trunk held by a set of belts. The shoulder and elbow joints were free and the wrist was encased in a brace. They were instructed to draw with an electric pen as fast and accurately as possible a series of geometric figures on a graphic tablet interfaced with a microcomputer. The geometric figures were triangles and squares of different amplitudes ( 24 and $48 \mathrm{~cm}$ perimeter) and were marked on the graphic tablet only by the vertices. After a few practice movements 15 single trials for each geometric figure were collected.

The $\mathrm{x}$ and $\mathrm{y}$ coordinates of the movements were directly obtained by the computer activated by the electric pen. The electromyographic activity of pectoralis major, posterior deltoid, biceps and triceps muscles was recorded by means of surface electrodes placed over the belly of the muscles, full wave rectified and integrated. The EMG activities and the $x$ and $y$ coordinates of the movements were recorded on a photographic paper and stored in a magnetic tape (Honeywell $5600 \mathrm{c}$ ). The accuracy of the movements at the vertices of the geometric figures was computed measuring the distances between the targets and the actual position reached by the subjects. In addition, the area of accuracy at each vertex of the figure was delimited including all the vertices reached by the subjects during the execution of 15 trials. The time necessary to draw the full figures (movement time) and the time spent at each vertex (pause) were measured. The number of the EMG bursts in the four muscles was measured by visual inspection. Student's $t$ test (unpaired) was used to analyse the data. 


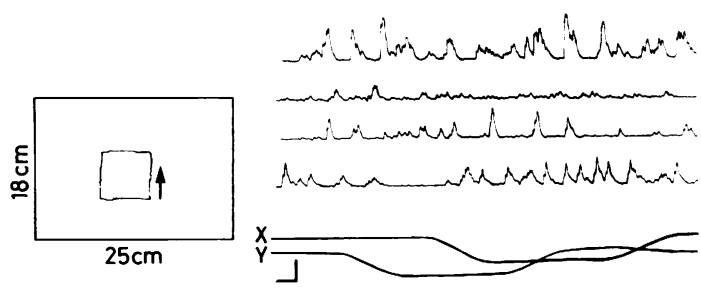

Fig 1 Execution of a square of $24 \mathrm{~cm}$ perimeter in a patient with Parkinson's disease. On the left: trajectory of the movement (small square); the arrow indicates the starting position and the direction of the movement. On the right: (from top to bottom) EMG activity of biceps, triceps, pectoralis major, posterior deltoid muscles and $x$ and $y$ coordinates of the movement. A single trial is shown. Calibration: $100 \mathrm{~ms}, 0 \cdot 2 \mathrm{mV}$.

P; Parkinsonians

\section{N: Normals}
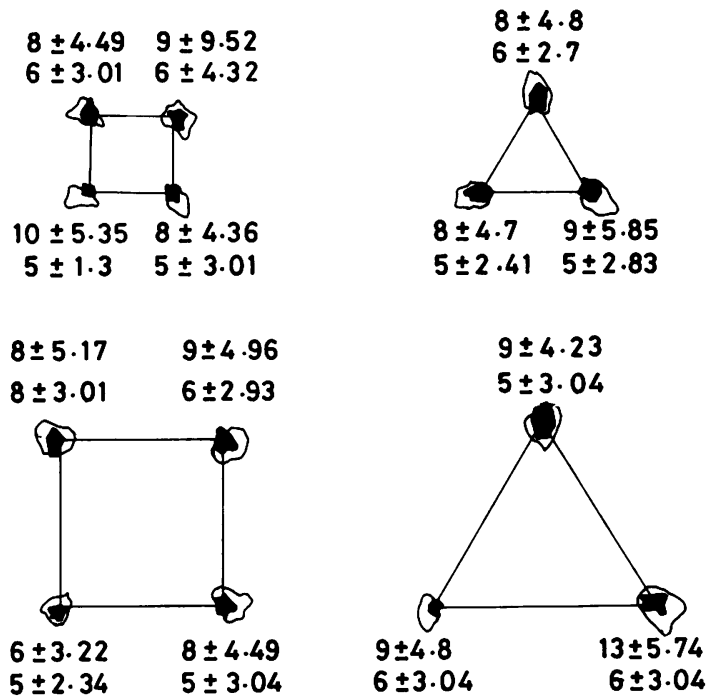

Fig 2 Accuracy of movements in normal subjects and in patients with Parkinson's disease. The area of accuracy at each vertex of the geometric figure was delimited including all the vertices reached by the subjects during the execution of 15 trials. Black areas represent the area of accuracy of Parkinsonian patients; white areas the same for normal subjects. The number represents the mean $\pm 1 S D$ of the distances (in $\mathrm{mm}$ ) between the requested vertices and the vertices actually reached by the subjects; independently of the spatial distributions of the movements.

\section{Results}

The Parkinsonian patients were able to draw accurately geometric figures of different size and shape (fig 1). The vertices of the figures were reached with more

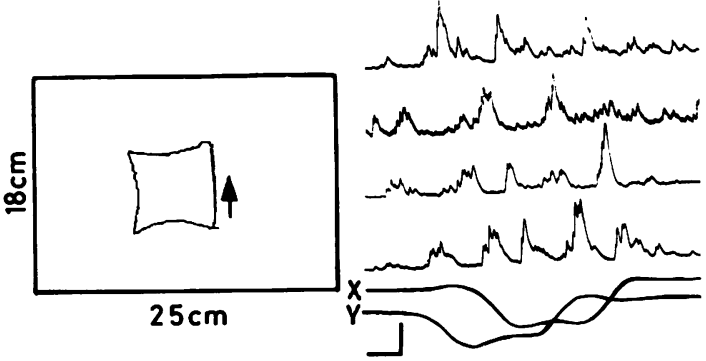

Fig 3 Execution of a square of $24 \mathrm{~cm}$ perimeter in a normal subject. On the left: trajectory of the movement (small square); the arrow indicates the starting position and the direction of the movement. On the right: (from top to bottom) EMG activity of biceps, triceps, pectoralis major, posterior deltoid muscles (from top to bottom) and the $x$ and $y$ coordinates of the hand movement are shown. A single trial is shown. Calibration: $100 \mathrm{~ms}, 0.2 \mathrm{mV}$.

accuracy than that observed in the normal subjects (fig 2). There was also less variability and less overshooting than normal subjects.

The segments of the triangles and squares were executed with trajectories which were roughly straight in the patients (fig 1) and more curvilinear in the normal subjects (fig 3 ).

The time employed to draw the figures was prolonged in comparison with normal subjects (table 1), and the duration of the pauses at the vertices was also longer (table 2).

There were no differences in accuracy and velocity of performance between tremulous and nontremulous Parkinsonians.

The electromyographic activity was characterised, in normal controls, by a pattern of EMG bursts in the agonist and antagonist muscles whether the emphasis was on speed or accuracy (fig 3); the EMG pattern was usually characteristic and stereotyped for each figure (triangle and square). In contrast, patients with Parkinson's disease showed a larger number of EMG bursts (fig 1, table 3 ) not correlated with the number of sides of the geometric figure.

When normal subjects were asked to be more accurate, they could slow down the movement and match

Table 1 Times (in ms) to draw the full geometrics figures. Data are means $\pm 1 S D .(p<0.001)$

\begin{tabular}{lll}
\hline & Normals & Patients \\
\hline Triangles (perimeter) & $582 \cdot 2 \pm 72 \cdot 8$ & $1341 \cdot 1 \pm 113 \cdot 1$ \\
$24 \mathrm{~cm}$ & $634.6 \pm 52 \cdot 2$ & $1590 \cdot 5 \pm 100 \cdot 1$ \\
$48 \mathrm{~cm}$ & $762.3 \pm 78 \cdot 3$ & $1790.5 \pm 138 \cdot 7$ \\
Squares (perimeter) & $4 \mathrm{~cm}$ & $2126 \cdot 1 \pm 143 \cdot 7$ \\
$48 \mathrm{~cm}$ & $835.8 \pm 139 \cdot 5$ & \\
\hline
\end{tabular}


Table 2 Pauses (in $\mathrm{ms}$ ) at the vertices of the geometrics figures. In the first and third columns are shown the pauses for each geometric figures (2 for the triangle and 3 for the square). In the second and fourth columns are shown the mean pauses. Data are the means $\pm 1 S D$. All the differences were significant. $(p<0 \cdot 001)$.

\begin{tabular}{|c|c|c|c|c|}
\hline & \multicolumn{2}{|l|}{ Normal } & \multicolumn{2}{|l|}{ Patients } \\
\hline & & Mean & & Mean \\
\hline \multicolumn{5}{|l|}{ Triangles (perimeter) } \\
\hline $24 \mathrm{~cm}$ & $\begin{array}{l}72.5 \pm 24.7 \\
56.9 \pm 22.1\end{array}$ & $64 \cdot 3 \pm 11 \cdot 6$ & $\begin{array}{l}188.7 \pm 75.9 \\
133.3 \pm 70.1\end{array}$ & $160.9 \pm 39.4$ \\
\hline $48 \mathrm{~cm}$ & $\begin{array}{l}58.5 \pm 26.8 \\
62.9 \pm 24.3\end{array}$ & $60 \cdot 3 \pm 2 \cdot 6$ & $\begin{array}{l}153.7 \pm 105.1 \\
134.7 \pm 75.0\end{array}$ & $144 \cdot 1 \pm 13 \cdot 5$ \\
\hline \multicolumn{5}{|l|}{ Squares (perimeter) } \\
\hline $24 \mathrm{~cm}$ & $\begin{array}{l}77 \cdot 3 \pm 31 \cdot 0 \\
89 \cdot 0 \pm 44 \cdot 0 \\
57 \cdot 0 \pm 26.0\end{array}$ & $74 \cdot 5 \pm 16 \cdot 1$ & $\begin{array}{l}186 \cdot 2 \pm 44 \cdot 0 \\
155 \cdot 1 \pm 38 \cdot 8 \\
165 \cdot 0 \pm 41\end{array}$ & $168.8 \pm 15.9$ \\
\hline $48 \mathrm{~cm}$ & $\begin{array}{l}75.3 \pm 42.9 \\
89.1 \pm 36.0 \\
80.0 \pm 44.5\end{array}$ & $81 \cdot 5 \pm 6 \cdot 9$ & $\begin{array}{l}154.5 \pm 59.4 \\
140.8 \pm 57.0 \\
159.3 \pm 35.8\end{array}$ & $151.6 \pm 9.7$ \\
\hline
\end{tabular}

Table 3 Numbers of EMG bursts in all the muscles (biceps,triceps, pectoralis major, posterior deltoid). Data are the means $\pm 1 S D . p<0.001$.

\begin{tabular}{lll}
\hline & Normal subjects & Patients \\
\hline Triangles (perimeter) & & \\
$24 \mathrm{~cm}$ & $3.35 \pm 1.21$ & $6.15 \pm 3.21$ \\
$48 \mathrm{~cm}$ & $3.38 \pm 0.84$ & $6.87 \pm 3.05$ \\
Squares (perimeter) & & \\
$24 \mathrm{~cm}$ & $4.15 \pm 1.27$ & $6.02 \pm 3.73$ \\
$48 \mathrm{~cm}$ & $4.03 \pm 1.01$ & $7.49 \pm 3.33$ \\
\hline
\end{tabular}

the accuracy of Parkinsonian patients. The velocity of performance was in this case not significantly different from Parkinsonian patients; the trajectory tended to become rectilinear and the number of EMG bursts increased. When Parkinsonian subjects were urged to speed up and perform their trajectory faster, no improvement of the velocity of performance could be obtained.

\section{Discussion}

The Parkinsonian patients were able to reach accurately and with roughly straight trajectories the vertices of a geometric figure. In addition, the variability of the end points was less than in normal subjects. The tendency to move the hand along straight pathways has been described in normal subjects during the execution of ballistic arm movements ${ }^{56}$; when the velocity of the movements increased the trajectory assumed a more curvilinear shape, and the variability at the end points increased. ${ }^{6}$ Thus the tendency to follow straight lines and the relative constancy at the end points of Parkinsonian patients may be due to the slowness of the movement. The time employed by the patients to draw the figures was prolonged in comparison to normal subjects.
The movement duration included the time necessary to cover the trajectory (movement time), ${ }^{13}$ and the delay at the vertices (pause). This last value can be split into reaction time, time for planning and time for running motor programmes. The movement time was significantly increased in Parkinsonian patients. The EMG bursts did not show the usual stereotyped pattern of agonist-antagonist discharge, the number of bursts was increased and not correlated with the number of sides of the geometric figure. This finding is similar to that reported during elbow ${ }^{2}$ and thumb movements ${ }^{3}$ at a single joint.

The pauses were also increased. It is unlikely that the pause prolongation may be due to an abnormal reaction time, since the subjects were requested to draw the figures without stopping at the vertices.

The ability to plan a motor programme seems intact also in the patients with Parkinson's disease. The relative timing of activity in the agonist and antagonist muscles is normal ${ }^{8}$ and the size and duration of the first agonist burst is normally modulated during movements of different amplitudes and loads (unpublished observations). Evarts $\mathrm{et} \mathrm{al}^{7}$ examining simple and choice reaction times concluded that there was no abnormality in formulating the central motor programme and Day et $a l^{9}$ and Bloxham et $a l^{10}$ have shown that Parkinsonian patients are capable of predictive motor behaviour.

Our hypothesis is that in Parkinson's disease the prolonged pause at the vertices reflects a difficulty in running motor programmes, particularly when it is necessary to switch from one programme to another. In fact, at the various positions of the upper limbs, the shoulder and arm muscles need to be activated in a different order and combination. In addition, Schwab et a ${ }^{11}$ have demonstrated in Parkinsonian patients the inability to execute simultaneously two motor acts such as squeezing a sphygnomanometer bulb with one 
hand and drawing a triangle with the other. More recently, Marsden ${ }^{8}$ has drawn attention to the fact that patients with Parkinson's disease cannot perform repetitive, sequential and concurrent motor actions and has proposed that this depends on inability to execute automatically learned motor plans.

In conclusion a difficulty in performing complex fast arm movements involving two joints is present in Parkinson's disease. The first agonist burst which normally provides the impulsive force for the movement is inadequate and followed by compensatory multiple bursts, and this slows down the execution of a simple ballistic movement. The slowing is such that the movement cannot any more be considered ballistic. In fact the velocity of execution allows corrections by ongoing activity. In addition while normal subjects can select between different motor strategies during complex trajectories, Parkinsonians can only utilise slower and more accurate ones. The delay between successive segments of complex trajectories is increased suggesting that there is also a difficulty in switching from one motor programme to the next. Our hypothesis is consistent with the conclusions reached by Bloxham et al $^{10}$ that in Parkinson's disease there is a difficulty in selecting and initiating motor plans.

We thank Mr Guido De Vito for technical assistance. This research was supported by the Italian Research Council (CNR). Dr JC Rothwell, Dr BL Day and
Prof $\mathbf{R}$ Benecke made useful comments about the manuscript.

\section{References}

${ }^{1}$ Flowers KA. Visual closed loop and open loop characteristics of voluntary movement in patients with Parkinsonism and intention tremor. Brain 1979;99:269-310.

${ }^{2}$ Hallett M, Khoshbin C. A physiological mechanism of bradykinesia. Brain 1980;103:301-14.

${ }^{3}$ Berardelli A, Rothwell JC, Day BL, Marsden CD. Movements not involved in posture are abnormal in Parkinson's disease. Neurosci Lett 1984;47:47-50.

${ }^{4}$ Dick JPR, Cowan JMA, Day BL, et al. The corticomotoneurone connection is normal in Parkinson's disease. Nature 1984;304:183-7.

${ }^{5}$ Morasso P. Spatial control of arm movements. Exp Brain Res 1981;42:223-7.

${ }^{6}$ Accornero N, Berardelli A, Argenta M, Manfredi M. Two joint ballistic arm movements. Neurosci Lett 1983;46:91-5.

${ }^{7}$ Evarts EV, Teravainen H, Calne DB. Reaction time in Parkinson's disease. Brain 1981;104:167-86.

${ }^{8}$ Marsden CD. The mysterious motor function of the basal ganglia. Neurology (NY) 1982;32:514-39.

${ }^{9}$ Day BL, Dick JPR, Marsden CD. Patients with Parkinson's disease can employ a predictive motor strategy. $J$ Neurol Neurosurg Psychiatry 1984;47:1299-306.

${ }^{10}$ Bloxham CA, Mindel TA, Frith CD. Initiation and execution of predictable and unpredictable movements in Parkinson's disease. Brain 1984;107:371-84.

${ }^{11}$ Schwab RS, Chafetz ME, Walker S. Control of two simultaneous voluntary motor acts in normals and parkinsonism. Arch Neurol Psychiatry 1954;72:591-8. 